\title{
The Churchwarden Accounts of Zoutleeuw's Church of Saint Leonard
}

The edition of the churchwarden accounts (De Mecheleer 1997a) suffers from several inaccuracies, for which reason a full review is offered here. Firstly, the account published as KR 1405 is in fact not a fragmentary churchwarden account of Saint Leonard's church, but rather a full account of Zoutleeuw's Capella clericorum. The edition does not include the first part of KR 15O2, nor does it include KR 1510 , KR 1572, or the official version of KR 1598. Furthermore, the two undated accounts can be identified as KR 1508 and KR 1510, respectively. These identifications are corroborated by data provided in the accounts themselves that match their respective preceding and subsequent accounts. Moreover, the first page of KR 1510 was found elsewhere, showing the same perforations and damages, and was written in the same hand. Lastly, the account published as KR 159 o is actually KR 156 o.

Unless otherwise noted, all original documents are in the Rijksarchief Leuven (RAL), collection Kerkarchief Brabant (KAB). These are either draft versions of the accounts compiled during the administrative year, or the official transcripts made for approval at its closing, which in some cases exist in the form of a contemporary second copy. In the table below, 'Account' refers to the version referred to throughout the text. Known copies or draft versions are inventoried under 'Copy' and 'Draft'. When only a draft version is known, reference is made to this version. The accounts in RAL, KAB, nos. 1218, 1222 and 1223 are unbound, and not foliated throughout. RAL, KAB, nos. $385^{21}, 3^{8} 5^{22}$ and $385^{23}$ are individual accounts.

Further remarks:

- It is unclear what the precise nature of each of the copies of KR 1453 are.

- Some of the accounts are damaged and/or incomplete: KR 1458; KR 1465; KR 1474; KR 1509; KR 1515; KR 1520; KR 1538; KR 156o; KR 1569; KR 1572; KR 1577; KR 159o; KR 1591; KR 1599.

- KR 1538 is incomplete, but although the title page is missing, its dating is corroborated by the data in the account.

- KR $155^{2}$ has been bound in the wrong order.

(C) RUBEN SUYKERBUYK, 2020 | DOI:10.1163/9789004433106_013

This is an open access chapter distributed under the terms of the CC BY-NC-ND 4.0 license. 


\begin{tabular}{|c|c|c|c|}
\hline KR & Account & Copy & Draft \\
\hline $145^{2}$ & 1214 , fols. $7-26 \mathrm{v}$ & & \\
\hline 1453 & 1214 , fols. $27-45^{v}$ & 1214 , fols. $46-74 \mathrm{~V}$ & 1214 , fols. $75^{-92 \mathrm{~V}}$ \\
\hline 1455 & 1214 , fols. $93^{-113 v}$ & 1214 , fols. $114^{-133 v}$ & \\
\hline $145^{6}$ & 1214 , fols. $134-176 \mathrm{v}$ & & \\
\hline 1457 & 1214 , fols. $177^{-196 v}$ & & \\
\hline $145^{8}$ & & & 1238 , fols. $117^{-146 v}$ \\
\hline 1459 & 1214 , fols. $197^{-21} 3^{v}$ & & \\
\hline 1460 & 1214 , fols. $234-254 \mathrm{~V}$ & 1214 , fols. $214^{-233 v}$ & \\
\hline 1463 & 1214 , fols. $255^{-272 \mathrm{~V}}$ & 1214 , fols. $273^{-291 v}$ & \\
\hline 1464 & & & 1214 , fols. $292-316 \mathrm{v}$ \\
\hline 1465 & 1214 , fols. $317-333 \mathrm{~V}$ & & \\
\hline 1466 & 1214 , fols. $35^{1-370 v}$ & 1214 , fols. $334-35$ ov & \\
\hline 1468 & 1215 , fols. $1-15 \mathrm{~V}$ & & \\
\hline 1469 & 1215 , fols. $32-49 v$ & 1215 , fols. $16-31 \mathrm{v}$ & \\
\hline 1471 & 1215 , fols. $5^{0-69 v}$ & 1215 , fols. $70-84 \mathrm{~V}$ & \\
\hline 1472 & 1215 , fols. $85^{-97 v}$ & 1215 , fols. $98-112 \mathrm{v}$ & \\
\hline 1473 & 1215 , fols. $113^{-125 \mathrm{~V}}$ & & 1238 , fols. $149^{-16} 5^{v}$ \\
\hline 1474 & 1215 , fols. $153^{-169 v}$ & & \\
\hline 1476 & 1215 , fols. $188-206 \mathrm{v}$ & 1215 , fols. $170-187 \mathrm{~V}$ & \\
\hline 1477 & 1215 , fols. $207-227 \mathrm{~V}$ & & \\
\hline 1478 & 1215 , fols. $228-248 v$ & & \\
\hline 1479 & 1215 , fols. $249-272 \mathrm{~V}$ & & \\
\hline 148 o & 1216 , fols. $19-33 \mathrm{v}$ & 1216 , fols. $1-18 v$ & \\
\hline 1481 & 1216 , fols. $54-67 \mathrm{v}$ & 1216 , fols. $34-53 \mathrm{v}$ & \\
\hline 1482 & 1216 , fols. $80-100 v$ & & \\
\hline 1483 & 1216 , fols. $101-120 \mathrm{~V}$ & & \\
\hline 1484 & 1216 , fols. $121-146 \mathrm{v}$ & & 1238 bis, fols. $45^{2}-488 v$ \\
\hline 1485 & 1216 , fols. $175^{-201 v}$ & 1216 , fols. $147^{-174 v}$ & \\
\hline 1486 & 1216 , fols. $231-25^{8 v}$ & 1216 , fols. $203^{-2} 3^{\circ}$ & \\
\hline 1487 & 1216 , fols. $285^{-309 v}$ & 1216 , fols. $261-283 v$ & \\
\hline 1489 & 1216 , fols. $310-329 \mathrm{v}$ & & \\
\hline 1490 & 1217 , fols. $25^{-48 v}$ & 1217 , fols. $1-24 \mathrm{~V}$ & \\
\hline 1491 & 1217 , fols. $49^{-69 v}$ & & \\
\hline 1492 & 1217 , fols. $70-101 \mathrm{v}$ & & \\
\hline 1493 & 1217 , fols. $102-124 \mathrm{~V}$ & & \\
\hline 1495 & 1217 , fols. $126-149 v$ & & \\
\hline 1496 & 1217 , fols. $15^{1-176 v}$ & & \\
\hline 1497 & 1217 , fols. $179-204 \mathrm{~V}$ & & \\
\hline
\end{tabular}


(cont.)

\begin{tabular}{|c|c|c|c|}
\hline KR & Account & Copy & Draft \\
\hline 1498 & 1217 , fols. $206-233 v$ & 1218 & \\
\hline $15^{\circ 0}$ & 1218 & & \\
\hline 1502 & & & 1238 , fols. $240-276 \mathrm{v}$ and 1238 bis, $416-45^{1 \mathrm{v}}$ \\
\hline 1503 & 1218 & & 1218 \\
\hline 1504 & 1218 & & 1218 \\
\hline 1505 & 1218 & & \\
\hline 1506 & 1218 & & \\
\hline 1507 & 1218 & & Possibly 1238 bis, fols. $559-564 \mathrm{v}$ and $625^{-656 \mathrm{v}}$ \\
\hline 1508 & 1238 , fols. $277-302 \mathrm{v}$ & & $\begin{array}{l}\text { Possibly } 1238 \text { bis, fols. } 559-564 \mathrm{v} \text { and } 625^{-656 v \text {, or }} \\
1238 \text { bis, fols. } 565^{-586 v}\end{array}$ \\
\hline 1509 & 1218 & 1218 & 1238 , fol. 101 and 1238 bis, fols. $587-624 \mathrm{~V}$ \\
\hline 1510 & $\begin{array}{l}1238, \text { fol. } 103 \text { and } \\
1238 \text { bis, fols. } 565^{-586 v}\end{array}$ & & \\
\hline 1511 & 1218 & & \\
\hline $15^{15}$ & 1218 & $123^{8}$, fols. $73^{-100 v}$ & \\
\hline $15^{16}$ & 1218 & & \\
\hline 1520 & 1219 , fols. $1-27$ & 1219 , fols. $28-53 \mathrm{v}$ & \\
\hline $15^{23}$ & 1219 , fols. $55^{-76 \mathrm{v}}$ & & 1219 , fols. $77^{-107 v}$ \\
\hline $15^{25}$ & 1219 , fols. $108-125$ & & \\
\hline $153^{\circ}$ & 1219 , fols. $129-162 \mathrm{~V}$ & & \\
\hline 1533 & 1219 , fols. $164-196 \mathrm{v}$ & & \\
\hline 1534 & 1218 & & \\
\hline 1538 & & & 1238 bis, fols. $348-391 \mathrm{~V}$ \\
\hline 1540 & 1219 , fols. $197^{-227 V}$ & & \\
\hline 1542 & 1219 , fols. $229^{-252 \mathrm{~V}}$ & & \\
\hline 1547 & 1219 , fols. $253^{-29 o v}$ & & \\
\hline 1548 & 1219 , fols. $291-329 v$ & & \\
\hline 1549 & 1219 , fols. $33^{0}-368 v$ & & \\
\hline $155^{\circ}$ & 1220 , fols. $1-36 \mathrm{v}$ & & \\
\hline $155^{1}$ & 1220, fols. $37-7 \mathrm{ov}$ & & \\
\hline $155^{2}$ & 1220 , fols. $71-121 \mathrm{~V}$ & & \\
\hline 1554 & 1220, fols. $122-181 \mathrm{~V}$ & & \\
\hline 1555 & 1220 , fols. $182-228 \mathrm{v}$ & & \\
\hline 1556 & 1220 , fols. $229-278 v$ & & \\
\hline 1557 & 1220 , fols. $279-320 v$ & & \\
\hline 1559 & 1220, fols. $321-366 \mathrm{v}$ & & \\
\hline 156 o & 1221, fols. $291-316 \mathrm{v}$ & & \\
\hline
\end{tabular}


(cont.)

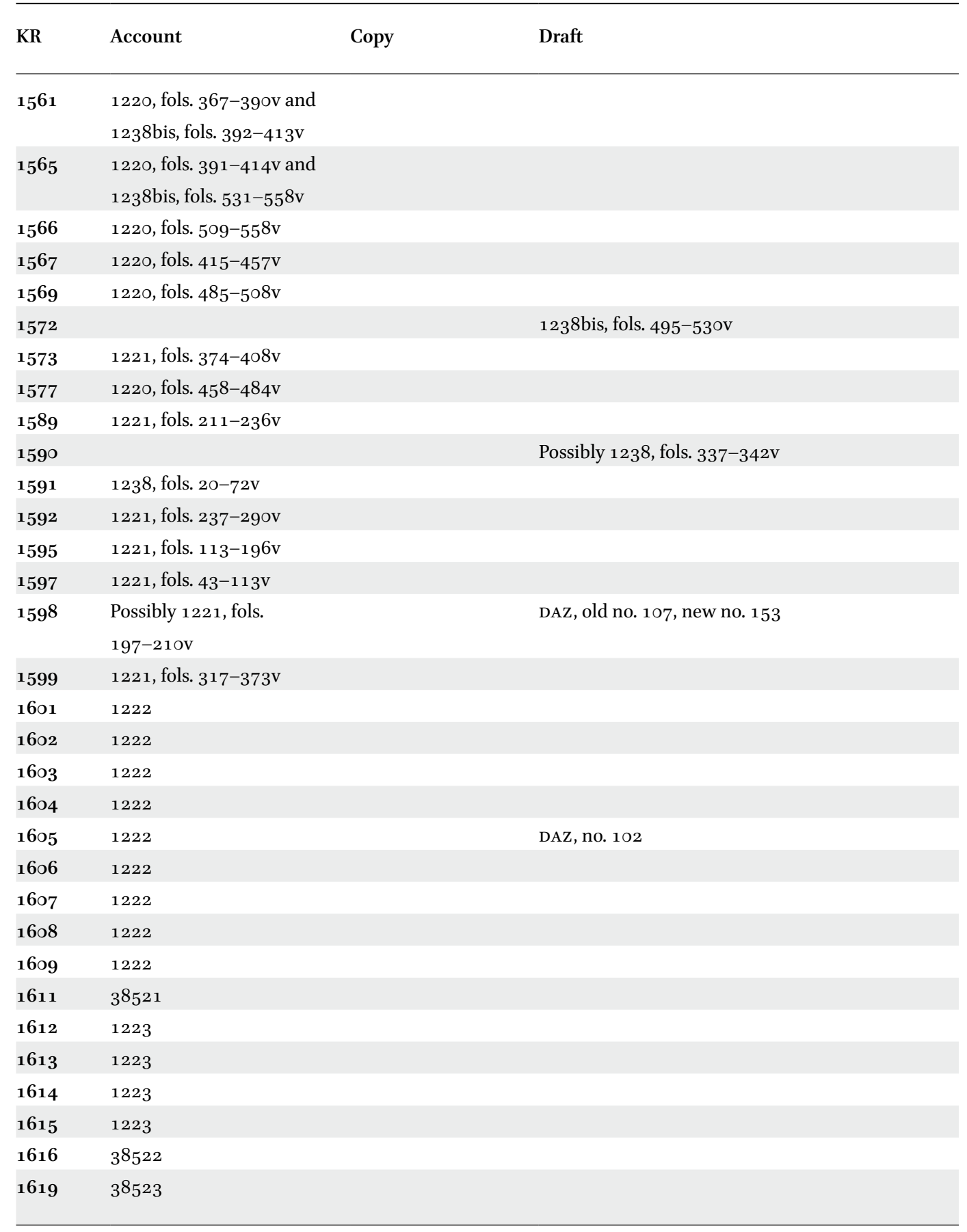

